\title{
The Banzhaf value for cooperative and simple multichoice games
}

\author{
Josep Freixas*
}

April 29, 2019

\begin{abstract}
This article proposes a value which can be considered an extension of the Banzhaf value for cooperative games. The proposed value is defined on the class of $j$-cooperative games, i.e., games in which players choose among a finite set of ordered actions and the result depends only on these elections. If the output is binary, only two options are available, then $j$-cooperative games become $j$-simple games. The restriction of the value to $j$-simple games leads to a power index that can be considered an extension of the Banzhaf power index for simple games. The paper provides an axiomatic characterization for the value and the index which is closely related to the first axiomatization of the Banzhaf value and Banzhaf power index in the respective contexts of cooperative and simple games.
\end{abstract}

Key words: An extension of the Banzhaf value; Axiomatic characterization of values; Multichoice games; UNSC voting system; Grading

JEL Class.: C71, D72.

\section{Introduction}

The Banzhaf power index is possibly the most-recognized measure for evaluating the a priori power of players in a simple game. The index was independently proposed by three authors: Penrose [19], Banzhaf [1] and Coleman [5]. A little later Owen [17] extended the power index to a value for cooperative games, which is known as the Banzhaf value.

Players only have two possible actions in the contexts of cooperative games and simple games. It is natural, therefore, to consider more general models in which players have a greater number of allowed ordered actions for cooperative games or several degrees of compliance in simple games.

A multi-choice game, considered by Hsiao and Raghavan [15, 16], is a generalization of a cooperative game in which each player has several ordered activity levels in the sense that, for every pair of different actions one action carries more 'weight' than the other action. The last level of activity is reserved for players without any activity. Cooperative games form a subclass of the class of multi-choice games.

*The author is with the Universitat Politècnica de Catalunya (Campus Manresa), in the Department of Mathematics; e-mails: josep.freixas@upc.edu, postal address: EPSEM, Avda. Bases de Manresa, 61-73, E-08242 Manresa, Spain. 
Extensions of simple games are mainly proposed in [9] for voting games including abstention as an intermediate vote between 'yes' and 'no', and in [13] a more general model was proposed, that of $(j, k)$-simple games, a class of voting games in which voters may choose any of $j$ ordered levels of approval and $k$ stands for the number of possible aggregated ordered results. The work in [13] provides a notion of weighted game endorsed by characterizations of the property of traderobustness. If $k=2$ the output has only two choices and the game is called $j$-simple game.

In this paper we propose a value that extends the Banzhaf value to $j$-cooperative games and to $j$-simple games, where $j$-cooperative games is a slightly different more convenient adaptation of multi-choice games, because its restriction to the binary case for the output gives rise to $j$-simple games. As shown below the proposed value gives an a priori valuation for each player in the game.

The rest of the paper is organized as follows. Some examples of $j$-cooperative games are presented in the remainder of this section. Section 2 introduces some formal preliminaries, the contexts of $j$-cooperative games and $j$-simple games, and the definition of a value in these contexts that extends the Banzhaf value for cooperative games. The main Section 3 provides an axiomatization for the value. Examples are revisited in Section 4 and the Conclusion ends the paper in Section 5.

\subsection{Some examples}

In this subsection, we present some examples to illustrate the versatility of the kind of games we consider. In describing these examples we use some intuitive terminology which is concisely defined in next section. A value that captures the idea of the Banzhaf value for cooperative games is proposed in Section 2 for a more general context.

Example 1.1 (A team of workers) A team of three workers have to perform a task. All three can carry out their task at three different levels: full involvement, medium involvement and lack of involvement. Only one of them, called $a$, is qualified to operate a machine that is essential to achieve a satisfactory execution of the work to be done. The other two workers, called $b$ and $c$, play a symmetrical role and also turn out to be indispensable together and a lack of involvement on the part of the two would be fatal for the execution of the task. Other combinations for these two workers with at least a medium involvement by worker a lead to more or less satisfactory results depending on the degree of involvement for these two workers. Full involvement by the three suppose a win of 4 monetary units. The following characteristic function specifies the gain for all combinations.

$$
v(S)= \begin{cases}4-\left|S_{2}\right|-2\left|S_{3}\right| & \text { if } a \in S_{1} \\ \max \left\{0,\left|S_{1}\right|-\left|S_{3}\right|\right\} & \text { if } a \in S_{2} \\ 0 & \text { if } a \in S_{3}\end{cases}
$$

where $S=\left(S_{1}, S_{2}, S_{3}\right)$ and $S_{1}$ contains the workers with full involvement, $S_{2}$ contains the workers with an intermediate involvement, and $S_{3}$ contains the rest of workers with the lowest level of involvement.

If we do not have any information about workers attitude, how should the total gain be distributed among them?

Example 1.2 (Grading) The evaluation of a subject consists of three independent tests, $a, b$ and $c$. Each test is rated with an integer mark in between 1 and 7, both included. To pass the subject requires that: 
i) the sum of the three grades obtained exceeds half of the possible points,

ii) a minimum grade of 3 is required for the test $a$, and

iii) a minimum of 4 is required for the average of tests $b$ and $c$.

The question we face is: what is the a priori importance of each test for the evaluation of the subject? Alternatively, consider the same question if instead, each test is rated with an integer mark in between 1 and 6 and the minimum average of 4 required in condition iii) is replaced by 3.5 .

Example 1.3 (The UNSC voting system) As noted by [10] the United Nations Security Council (UNSC) can be modeled as a 3-simple game: a resolution is approved if there are at least nine members in favor and permanent members are not against it. This means that also if some of the permanent members abstain, without explicitly imposing the veto, a resolution can be carried on. The resulting game $v$ has 15 players, with the subset $P$ of the five permanent member, and a tripartition $S=\left(S_{1}, S_{2}, S_{3}\right)$ is winning (i.e., $\left.v(S)=1\right)$ if and only if

$$
\left|S_{1}\right| \geq 9 \text { and } S_{3} \cap P=\emptyset .
$$

where $S_{1}$ contains the members in favor of the resolution, $S_{3}$ the members against it, and $S_{2}$ the abstainers. For further discussion on this significant system see for example [6].

The UNSC is critical to global peace and security, yet more than twenty years of negotiations over its reform have proved fruitless, see in [14] a survey on several proposed reforms that have not been implemented. A minimal modification of the UNSC voting game is proposed here. It consists in just modifying the possibility of approval of a resolution if one permanent member is against it but all the other members are in favor of it. This means that for any permanent member $p \in P$, the five losing tripartitions $(N \backslash\{p\}, \emptyset,\{p\})$ of the current system convert into winning tripartitions, and this is the only difference between the current and the proposed UNSC voting system. The inclusion of these five tripartitions in the set of winning tripartitions prevents the permanent members to have veto-right, but this situation only occurs when the other fourteen countries agree to vote in favor of the resolution at hand.

\section{Preliminaries on $j$-cooperative games}

We formally introduce the class of games we deal with in this paper, the notions are mainly taken from [13].

Let $N$ be a finite set of players. A $j$-partition of $N$ is a collection of $j$ mutually disjoint subsets of $N, S_{1}, \ldots, S_{j}$ such that $\cup_{k=1}^{j} S_{k}=N$. Note that any $S_{i}$ may be empty. Any subset $S$ of $N$ is called a coalition and we denote its cardinality by $s$.

A $j$-partition describes a division of players among $j$ alternatives or $j$ levels of voting approval or $j$ possible actions or choices players can realize or choose. We assume that these $j$ different alternatives are ordered and convey that level 1 corresponds to the highest level of performance, while the last, level $j$, corresponds to the lowest level. Thus, players in $S_{1}$ are those who work at the highest level, while those in $S_{j}$ work at the lowest level of activity. In a voting context, voters in $S_{1}$ are those who vote for the highest level of approval, whereas those in $S_{j}$ are those who vote for the lowest level of approval. Thus, the convention chosen is ordinal rather than numerical. 
From now on we denote with $J^{N}$ the set of all $j$-partitions on $N$ endowed with an (strict) order from the first (highest) order of performance or activity to the last (lowest) one. Although we assume an order of the levels of activity, we do not do any assumption over the quantification of these levels. Thus, acting at the second level just means that such level of activity is lower than in level 1 but greater than in level 3 .

A partial order $\subseteq^{j}$ on the set $J^{N}$ is considered. If $S, T \in J^{N}$, then $S \subseteq \subseteq^{j} T$ means $S_{k} \subseteq^{j} \bigcup_{i=1}^{k} T_{i}$ for any $k=1, \ldots, j-1$. In words, $S$ is contained in $T$ if players in $T$ are working or voting in the same or in a higher level than in $S$. We use $S \subset^{j} T$ if $S \subseteq^{j} T$ and $S \neq T$. The $j$-partitions $\mathcal{N}=(\emptyset, \ldots, \emptyset, N)$ and $\mathcal{M}=(N, \emptyset, \ldots, \emptyset)$ are respectively the minimum and maximum for the order $\subseteq^{j}$.

A binary voting situation in which voters (we use the term voters instead of the term players in the voting context) can vote among several ordered alternatives can be formalized by a $(j, 2)$-simple game, i.e., voters can vote in $j$ different ordered ways to approve or reject a resolution and the aggregate output is binary. As previously said, we refer to $(j, 2)$-simple game as $j$-simple games throughout this article.

Definition 2.1 Let $N$ be a finite set and $J^{N}$ be the set of all totally ordered $j$-partitions on $N$. A $j$-simple game is a function $v: J^{N} \rightarrow\{0,1\}$ such that: i) it is monotonic: if $S \subset^{j} T$ then $v(S) \leq v(T)$; ii) $v(\mathcal{N})=0$ and $v(\mathcal{M})=1$.

We denote with $\mathcal{S} \mathcal{J}_{N}$ the space of all $j$-simple games on the finite set $N$. Note that $(2,2)$-simple games are simple games since for any bipartition $S=\left(S_{1}, S_{2}\right)$ the first component $S_{1}$ is identified with the set of 'yes'-voters and $S_{2}=N \backslash S_{1}$ with the set of 'no'-voters. Thus, any bipartition is in one-to-one correspondence with coalition $S_{1}$. Note also that $(3,2)$-simple games can be interpreted as ternary voting games, as considered by [9], if the first level of approval correspond to voting 'yes', the second level to abstain and the third level to voting 'no'.

The set of winning $j$-partitions of $v$ is denoted by $W(v)=\left\{S \in J^{N}: v(S)=1\right\}$ and the set of minimal winning $j$-partitions by $W^{m}(v)=\left\{S \in W(v): T \notin W(v)\right.$ if $\left.T \subset^{j} S\right\}$.

In any $j$-simple game the aggregated output set is binary and represented by $\{0,1\}$, where these two numbers have the respective meaning that the submitted proposal is either defeated or passed.

Definition 2.2 Let $N$ be a finite set and $J^{N}$ be the set of all totally ordered $j$-partitions on $N$. A $j$-cooperative game is a function $v: J^{N} \rightarrow \mathbb{R}$ such that $v(\mathcal{N})=0$.

We denote by $\mathcal{J}_{N}$ the space of $j$-cooperative games on the finite set $N$. Note that a 2 -cooperative game corresponds to a cooperative game in which the bipartition $S=\left(S_{1}, N \backslash S_{1}\right)$ is identified with the coalition $S_{1}$ formed by players who decide to cooperate.

The previous definition is almost equivalent to that of a multi-choice game as defined in $[15,16]$. A distinction is that in the multi-choice setting an input level is distinguished from the others and it is reserved for the lack of activity. In our context the last input level does not necessarily mean a total lack of activity and this becomes clear in the voting context, for $j$-simple games. For instance, for ternary voting games $[10]$ ( $j=3$ with three input choices: voting 'yes', 'abstain' or voting 'no') the last input level means voting against the submitted proposal, which would not be coherent with the assumption made in the multi-choice model. The inactivity level for a multichoice game could be interpreted as void vote or absence to the vote, which in voting is a very sensitive issue 
since in certain voting systems (for example with quorums see [13, 22]) an absence can be more harmful than a negative vote.

There are many interesting subclasses of cooperative games that can easily be extended to $j$-cooperative games for $j>2$. Here we just refer to monotonicity since the most of the modeled situations fulfill this property.

A $j$-cooperative game is monotonic, if for any pair of $j$-partitions $S$ and $T$, such that $S \subset^{j} T$ then $v(S) \leq v(T)$. the examples we consider are monotonic.

As we shall see $\mathcal{J}_{N}$ is a vectorial space of dimension $j^{n}-1$ and a basis formed by monotonic $j$-cooperative games is the one of unanimity games defined as:

$$
u_{S}(T)= \begin{cases}1, & \text { if } S \subseteq^{j} T \\ 0, & \text { otherwise }\end{cases}
$$

for all $j$-partition $S \neq \mathcal{N}$.

\subsection{A value for $j$-cooperative games}

Let us introduce the following notation. From a given $j$-partition $S$, we define the $j$-partition $S_{a \downarrow_{k}}$ in which player $a \in S_{1}$ has moved from the highest level of activity 1 to the inferior level $k(k>1)$.

$$
S_{a \downarrow_{k}}=\left(S_{1} \backslash\{a\}, \ldots, S_{k} \cup\{a\}, \ldots, S_{j}\right)
$$

for any $k=2, \ldots, j$. When necessary we identify $S_{a \downarrow_{1}}$ with $S$.

The idea we pursue with this definition is to consider a special type of marginal contributions for $j$-partitions in a given game $v$ :

$$
m_{k}(v, S, a)=v(S)-v\left(S_{a \downarrow_{k}}\right) \quad \text { if } \quad a \in S_{1}
$$

In next definition we propose a value for $j$-cooperative games inspired with the ideas of the Banzhaf value for cooperative games, [7]. The explicit formula for the proposed value depends on the marginal contribution $m_{j}(v, S, a)$.

Definition 2.3 (A value for $j$-cooperative games) For any $v \in \mathcal{J}_{N}$ and any player $a \in N$, the $\mathcal{B}$-value is defined as

$$
\mathcal{B}_{a}(v)=\frac{1}{j^{n-1}} \sum_{\substack{S \in J^{N} \\ a \in S_{1}}} m_{j}(v, S, a) .
$$

Its restriction to $j$-simple games is denoted here by $\mathfrak{b}$, and we then refer to the $\mathfrak{b}$-power index. It was already considered in [11] for an arbitrary number of inputs $j$, and for $j=3$ in $[21,20,12,18]$.

\section{An axiomatization for the value}

The first idea that comes to mind is whether Dubey and Shapley's classic axioms or the adaptation of them to $j$-cooperative games serve to characterize the considered value. This is the purpose of the paper. 
It is not difficult to verify that these axioms are met for the considered value and it is also simple to realize that these axioms are not enough to uniquely characterize it. In cooperative games the axioms of total power, equal treatment and that of null player determine the Banzhaf value on the unanimity games, which by induction and the axiom of additivity (or transfer for simple games) uniquely extend the value to the rest of games.

\subsection{Axioms naturally extended from cooperative games}

In the following, $\psi: \mathcal{J}_{N} \rightarrow \mathbb{R}^{n}$ is a value for $j$-cooperative games.

Equal Treatment (ET) The value $\psi$ satisfies equal treatment if for any $a, b \in N$ and game $v \in \mathcal{J}_{N}$ it holds:

$$
\psi_{a}(v)=\psi_{b}(v)
$$

if $a$ and $b$ are equivalent.

Two players $a$ and $b$ are equivalent if for every $S$ such that $\{a, b\} \subseteq S_{1}$ it holds $m_{k}(v, S, a)=$ $m_{k}(v, S, b)$ for all $k=2, \ldots, j$.

Null Player $(\mathrm{N})$ The value $\psi$ satisfies the null player axiom if given a null player $a$ in the game $v$, then

$$
\psi_{a}(v)=0
$$

Player $a$ is null in the $j$-cooperative game $v \in \mathcal{J}_{N}$ if $m_{j}(v, S, a)=0$ for all $S$ with $a \in S_{1}$.

Additivity (Ad) The value $\psi$ satisfies additivity if for any $v, w \in \mathcal{J}_{N}$

$$
\psi(v+w)=\psi(v)+\psi(w)
$$

Transfer $(\mathrm{T})$ The index $\psi$ satisfies transfer if for any $v, w \in \mathcal{S} J_{N}$

$$
\psi(v)+\psi(w)=\psi(v \wedge w)+\psi(v \vee w),
$$

where $(v \wedge w)(S)=\min \{v(S), w(S)\}$ and $(v \vee w)(S)=\max \{v(S), w(S)\}$ for all $S \in J^{N}$.

We remark that a stronger condition that can replace equal treatment in Theorem 3.5 is anonymity. The value $\psi$ satisfies anonymity if for all game $v \in \mathcal{J}_{N}$, any permutation $\pi$ of $N$ and any $a \in N$ it holds $\psi_{a}(v)=\psi_{\pi(a)}(\pi v)$, where $(\pi v)(S)=v(\pi(S))$.

The conjunction, $v \wedge w$, and the disjunction, $v \vee w$ verify the following properties:

a. $W(v \wedge w)=W(v) \cup W(w)$ and $W(v \vee w)=W(v) \cap W(w) ;$

b. if $W^{m}(v)=\left\{S^{1}, \ldots, S^{k}\right\}$ then $v=u_{S^{1}} \wedge \cdots \wedge u_{S^{k}}$;

c. given two unanimity games $u_{S}$ and $u_{T}$, then their conjunction is still a unanimity game and $u_{S} \wedge u_{T}=u_{Z}$ where the $j$-partition $Z$ is obtained from $S$ and $T$ as follows: $a \in Z_{i}$ if and only if either $a \in S_{i}$ and $a \in T_{h}$ with $h \leq i$ or $a \in T_{i}$ and $a \in S_{h}$ with $h \leq i$.

The next result is a simple check that is left to the reader. 
Lemma $3.1 \quad i)$ The $\mathcal{B}$-value for $j$-cooperative games satisfies the axioms of: equal treatment, null player and additivity.

ii) The $\mathfrak{b}$-power index for $j$-simple games satisfies the axioms of: equal treatment, null player and transfer.

Note that both $\mathcal{B}$ and the $\mathfrak{b}$-power index satisfy the transfer axiom because the sets of $j$ cooperative and $j$-simple games are respective lattices with the operations of conjunction and disjunction that have a maximal and a minimal element, see [8]. Moreover, the property of additivity is also verified by $\mathcal{B}$.

We propose two new axioms on unanimity games that together with the other three uniquely characterize the $\mathcal{B}$-value and the $\mathfrak{b}$-power index for $j$-cooperative games and $j$-simple games respectively.

\subsection{Two axioms on unanimity games}

Now we introduce two new axioms that concern unanimity games. Let $S$ be a $j$-partition with $a \in S_{1}$ and $u_{S}$ be the unanimity game of the $j$-partition $S$. In the first axiom below, it is understood that $u_{S_{a \downarrow 1}}=u_{S}$, i.e., as $a$ remains in the first level of approval, the $j$-partition $S$ remains invariant.

No loss when voting at a lower level $\mathrm{A}$ value or index $\psi$ satisfies the no loss (when voting at a lower level) axiom if for any unanimity game $u_{S}$ with $a \in S_{1}$ and $i<j-1$ it holds

$$
\psi_{a}\left(u_{S_{a \downarrow i}}\right)=\psi_{a}\left(u_{S_{a \downarrow}(i+1)}\right) .
$$

Note that the previous axiom only has sense for $j \geq 3$.

Let $S \neq \mathcal{N}$ be an arbitrary $j$-partition, the harmonic cardinality of $S$ by the importance of the inputs, $h(S)$, is defined as

$$
\sum_{i=1}^{j-1} \frac{s_{i}}{i}
$$

so that the importance of the input when voting is highlighted by the harmonic weights: the higher the level of approval, the greater the harmonic cardinal.

The structural decisiveness index of a $j$-simple game, natural extension of this notion for simple games $[5,3,4]$, is now defined as

$$
\delta(v)=\frac{|W(v)|}{j^{n}}
$$

and gives a measure in between 0 and 1 about the cost of passing decisions in the game, assuming uniform distribution over all $j$-partitions.

Note that if $S \subset^{j} T$ it holds $h(S)<h(T)$ and $\delta\left(u_{S}\right)>\delta\left(u_{T}\right)$.

Total power on unanimity games An index $\psi$ satisfies the total power on unanimity games if for any $j$-tripartition $S \neq \mathcal{N}$ it holds

$$
\sum_{a \in N} \psi_{a}\left(u_{S}\right)=j \cdot \delta\left(u_{S}\right) \cdot h(S)
$$


The last axiom expresses that the total power on any unanimity game is the product of:
a. the number of input alternatives $j$,
b. the structural decisiveness of the game $u_{S}$, and
c. the harmonic cardinality of $S$.

Different from what happens with the no loss axiom, this one is valid for $j \geq 2$.

The following consecutive trivial lemmas give sense to what we pursue, by its simplicity we omit their proofs that consist of checking that the $\mathcal{B}$-value (or $\mathfrak{b}$-power index when we are restricted to $j$-simple games) as defined in (1) satisfies some properties.

Proposition 3.2 Let $S \neq \mathcal{N}$ be a $j$-partition and $a \in S_{i}$, then both $\mathcal{B}$ and $\mathfrak{b}$ satisfy

$$
\psi_{a}\left(u_{S}\right)= \begin{cases}\frac{j}{i} \delta\left(u_{S}\right), & \text { if } i<j \\ 0, & \text { if } i=j\end{cases}
$$

Proof: In the game $u_{S}, S$ is the unique minimal winning $j$-partition. The rest of winning $j$ partitions in $u_{S}$ are obtained by shifting players from lower levels to upper levels. Thus, $\left|W\left(u_{S}\right)\right|$ depends only on the positions of the players in $S$ and it results $\left|W\left(u_{S}\right)\right|=\prod_{k=1}^{j} k^{s_{k}}$. Only the $j$-partitions $T$ with $a \in T_{1}, u_{S}(T)=1$ and $u_{S}\left(T_{a \downarrow j}\right)=0$ count for the Banzhaf value. Thus, $\mathcal{B}_{a}\left(u_{S}\right)=0$ if $a \in S_{j}$ because $a \in T_{1}$ and $u_{S}(T)=1$ implies $u_{S}\left(T_{a \downarrow j}\right)=1$, i.e., $m_{j}\left(u_{S}, T, a\right)=0$ for all $j$-partition $T$ with $a \in T_{1} \cap S_{j}$. Assume now, $a \notin S_{j}$, then,

$$
\mid\left\{T \in J^{N}: a \in T_{1}, a \in S_{i} \text { and } m_{j}\left(u_{S}, T, a\right)=1\right\} \mid=\frac{\left|W\left(u_{S}\right)\right|}{i}
$$

and therefore

$$
\mathcal{B}_{a}\left(u_{S}\right)=\frac{1}{j^{n-1}} \cdot \frac{\left|W\left(u_{S}\right)\right|}{i}=\frac{j}{i} \cdot \frac{\left|W\left(u_{S}\right)\right|}{j^{n}}=\frac{j}{i} \cdot \delta\left(u_{S}\right) .
$$

By applying Proposition 3.2 we derive the following consequences.

Corollary 3.3 Both $\mathcal{B}$ and $\mathfrak{b}$ satisfy the no loss axiom

$$
\psi_{a}\left(u_{S_{a \downarrow i}}\right)=\psi_{a}\left(u_{S_{a \downarrow}(i+1)}\right),
$$

with $a \in S_{1}$ and $i<j$.

Proof: By Proposition 3.2 it follows:

$$
\psi_{a}\left(u_{S_{a \downarrow i}}\right)=\frac{j}{i} \delta\left(u_{S_{a \downarrow i}}\right) \quad \text { and } \quad \psi_{a}\left(u_{S_{a \downarrow(i+1)}}\right)=\frac{j}{i+1} \delta\left(u_{S_{a \downarrow}(i+1)}\right)
$$


Corollary 3.4 Both $\mathcal{B}$ and $\mathfrak{b}$ satisfy the total power axiom on unanimity games.

Proof: By Proposition 3.2 it follows:

$$
\sum_{i=1}^{j-1} \frac{j}{i} \delta\left(u_{S}\right) s_{i}=\sum_{i=1}^{j-1} j \delta\left(u_{S}\right) \frac{s_{i}}{i}=j \delta\left(u_{S}\right) \sum_{i=1}^{j-1} \frac{s_{i}}{i}=j \delta\left(u_{S}\right) h(S)
$$

Thus, the axioms of equal treatment, null player, total power and no loss uniquely characterize the value on unanimity games, which by transfer (or also additivity for $\mathcal{B}$ ) extend to the rest of $j$-cooperative games or $j$-simple games as shown in the next result.

\subsection{An axiomatic characterization}

Theorem 3.5 Let $j \geq 3$.

i) A value $\psi$ on $j$-cooperative games satisfies equal treatment, null-player, total power on unanimity games, no loss for unanimity games and additivity axioms if and only if $\psi=\mathcal{B}$.

ii) A value $\psi$ on $j$-simple games satisfies equal treatment, null-player, total power on unanimity games, no loss for unanimity games and transfer axioms if and only if $\psi=\mathfrak{b}$.

Note that for $j=2$ (cooperative and simple games) the previous axiomatization remains valid by removing the no loss axiom.

Proof: The proof is organized in five parts:

1) We have seen, in Lemma 3.1 and Corollaries 3.3 and 3.4, that both $\mathcal{B}$ and $\mathfrak{b}$ satisfy the respective axioms.

2) We now prove that a value, which satisfies the four first axioms becomes uniquely determined on unanimity games.

Recall that $\mathcal{M}$ is the $j$-partition in which all voters choose the highest level of approval. Let $S \neq \mathcal{N}$. We consider a parameter which relates the $j$-partitions $\mathcal{M}$ and $S: d=\sum_{k=1}^{j}\left(n_{k}-s_{k}\right) \cdot(j-k)$. We will proceed by induction on unanimity games $u_{S}$ with a given common parameter $d$, which can be interpreted as the number of one step-down for players necessary to convert $\mathcal{M}$ into $S$.

Consider $u_{S}$. If $d=0$ then $S=\mathcal{M}$ and the value on the unanimity game $u_{\mathcal{M}}$ is uniquely determined by anonymity and total power on the unanimity game $u_{\mathcal{M}}$. If $d=1$ then there is a unique $a \in N$ such that $a \in S_{2}$ and $S_{1}=N \backslash\{a\}$. The value of $a$ in $u_{S}$ is determined by the no loss axiom, which coincides with the value of $a$ in $u_{\mathcal{M}}$, the value of players in $S_{1}$ is then determined by equal treatment and total power on the unanimity game $u_{S}$.

Assume the assumption is true for all games $u_{T}$ with a parameter $d$ for $T$. Let us prove the statement for all games $u_{S}$ with a parameter $d+1$ for $S$. The value in $u_{S}$ for players in $S_{j}$ is zero for the null-player axiom, the value in $u_{S}$ for players in $S_{i}$ for every $1<i<j$ coincide, by the no loss axiom, with the value these players have in $u_{T}$, where $T$ is obtained from $S$ by only 
changing the position of the involved player in $S_{i}$ to the immediate higher level of approval $i-1$. By induction hypothesis, such value in $u_{T}$ is uniquely determined so it is also in $u_{S}$. The value in $u_{S}$ of players in $S_{1}$ is then uniquely determined by equal treatment and total power on $u_{S}$. As we deal with $j$-simple games this proof is valid for both $\mathcal{B}$ and $\mathfrak{b}$.

3) The unanimity games form a basis of the vectorial space $\mathcal{J}_{N}$ of dimension $j^{n}-1$.

If $v$ is any $j$-cooperative game, then there exist $j^{n}-1$ real numbers $c_{S}$, which are uniquely determined for every $j$-partition $S \neq \mathcal{N}$ such that

$$
v=\sum_{S \subseteq^{j} \mathcal{M}} c_{S} u_{S}
$$

The existence and uniqueness of these coefficients follow by applying equation (2) to any arbitrary $j$-partition $R, R \neq \mathcal{N}$.

$$
v(R)=\sum_{S \subseteq^{j} \mathcal{M}} c_{S} u_{S}(R)
$$

Then it results a system of $j^{n}-1$ linear equations with $j^{n}-1$ unknowns, the numbers $c_{S}$ with $S \neq \mathcal{N}$. The resulting matrix, obtained by ordering the images of the characteristic function $v$ in lexicographic order ${ }^{1}$ is triangular superior, i.e. zeros under the diagonal, and all the numbers in the diagonal are ones.

Thus, the determinant is one and therefore the linear system is compatible determined. Thus, the coefficients $c_{S}$ are uniquely determined ${ }^{2}$. By additivity and Equation (2) the value of $\psi$ in an arbitrary game $v$ is determined by the value of $\psi$ on unanimity games.

4) By the transfer axiom the value is uniquely extended to the rest of $j$-cooperative games.

Let $W^{m}(v)=\left\{S^{1}, S^{2}, \ldots, S^{k}\right\}$. We proceed by induction on the number $k$ of minimal winning $j$-partitions. If $k=1$ the game is a unanimity game and therefore uniquely determined for $\psi$. Assume the hypothesis is true for all integer lower than $k$. As $\psi$ satisfies the transfer axiom we have:

$$
\psi(v)=\psi\left(u_{S^{1}}\right)+\psi\left(u_{S^{2}} \vee \cdots \vee u_{S^{k}}\right)-\psi\left(u_{S^{1}} \wedge u_{S^{1}} \wedge \cdots \wedge u_{S^{k}}\right)
$$

As the conjunction of unanimity games is a unanimity game it results

$$
\psi(v)=\psi\left(u_{S^{1}}\right)+\psi\left(u_{S^{2}} \vee \cdots \vee u_{S^{k}}\right)-\psi\left(u_{Z}\right) .
$$

where the $j$-partition $Z$ is such that $a \in Z_{i}$ if $i$ is the greatest level of approval to which $a$ belongs to in the $j$-partitions $S^{h}$ for $h=1, \ldots, k$.

The value $\psi$ is uniquely determined on $v$ since the number of minimal winning $j$-partitions in the games: $v, u_{S^{2}} \vee \cdots \vee u_{S^{k}}$ and $u_{Z}$ are lower than $k$.

5) By 1) $\mathcal{B}$ and $\mathfrak{b}$ satisfy the respective axioms stated. By 2) they are uniquely determined on unanimity games. By 3) $\mathcal{B}$ uniquely extends to the rest of $j$-cooperative games. By 4) $\mathfrak{b}$ uniquely extends to the rest of $j$-simple games.

\footnotetext{
${ }^{1} S$ precedes $T$ in the lexicographic order if for the smallest $p \in N$ such that $p \in S_{i}$ and $p \in T_{h}$ with $i \neq h$, it holds $i<h$.

${ }^{2}$ By solving the linear system of equations it can be seen that these coefficients are $c_{S}=\sum_{T \subseteq^{j} S}(-1)^{\sum_{i=1}^{j}\left(t_{i}-s_{i}\right)} v(T)$.
} 
It is very simple to verify the independence of these axioms for arbitrary values of $j$, as shown in [2] for the particular case of 3-simple games. The extensions of the examples used there for greater values of $j$ becomes trivial. Of course, when $j=2$ the value $\mathcal{B}$ for $j$-cooperative games coincides with the known Banzhaf value for cooperative games as considered by Owen [17].

\section{Examples}

Example 1.1 revisited It is a straightforward check that the $\mathcal{B}$ value is $(2,1,1)$, i.e., the qualified worker must receive twice as much as any of the other two.

Example 1.2 revisited By the equal treatment property the tests $b$ and $c$ have the same importance by $\mathcal{B}$. It is easy to check that in the former case $(j=7$ options, $a \geq 3$, and $b+c \geq 8)$ we obtain:

$$
\mathcal{B}_{a}=\frac{28}{49}<\frac{30}{49}=\mathcal{B}_{b}=\mathcal{B}_{c}
$$

For the second case ( $j=6$ options, $a \geq 3$, and $b+c \geq 7)$ we obtain:

$$
\mathcal{B}_{a}=\frac{21}{36}>\frac{20}{36}=\mathcal{B}_{b}=\mathcal{B}_{c}
$$

Thus, the rankings of $\mathcal{B}$ for players are different for the two assumptions.

Example 1.3 revisited Recall that for the UNSC voting system the winning tripartitions $S$ satisfy

$$
\left|S_{1}\right| \geq 9 \text { and } S_{3} \cap P=\emptyset .
$$

And for the proposed reform of the UNSC voting system the winning tripartitions $S$ satisfy

$$
\text { either } \quad\left|S_{1}\right| \geq 14 \quad \text { or } \quad 13 \geq\left|S_{1}\right| \geq 9 \text { and } S_{3} \cap P=\emptyset .
$$

In both models there are more than 14,3 million of tripartitions with a given member voting 'yes'. The difference between the two models is that the 5 tripartitions $(N \backslash\{p\}, \emptyset,\{p\})$ where $p$ is a permanent member, which are losing in the classical model become winning in the new one, which has two effects when computing $\mathfrak{b}$.

For a rotatory member the number of new winning tripartitions that convert into losing when the member changes her vote from 'yes' to 'no' are 5: $(N \backslash\{p\}, \emptyset,\{p\})$ for each permanent member $p$.

For a permanent member the number of new winning tripartitions that convert into losing when the member changes her vote from 'yes' to 'no' are 4: $(N \backslash\{p\}, \emptyset,\{p\})$ for any other permanent member $p$, but the winning tripartition $\mathcal{M}$ remain winning if $p$ changes her vote from 'yes' to 'no'.

Thus when computing the index $\mathfrak{b}$ for a permanent member in the reformed system, the numerator in Equation (1) is increased by 3 with respect to the numerator for the classical system, this increase for a rotatory member is 5 . The index $\mathfrak{b}$ is computed in [12] for the UNSC voting system resulting that the proportion of power between a permanent member and a rotatory member is $\mathfrak{b}_{p} / \mathfrak{b}_{r} \approx 2.04$ that hardly suffers variation for the reformed system. That is, permanent members could lose their right to veto without having their a priori Banzhaf power altered. 


\section{Conclusion}

The value proposed in this paper for $j$-cooperative games has ingredients to be a generalization of the Banzhaf value. Among the arguments supporting the value proposed here we can find the following: it is consistent in its particularization from $j$-cooperative games to $j$-simple games; it admits a simple explicit formula in terms of the characteristic function; it is supported by an axiomatic characterization which is close to the first characterization of the Banzhaf value in cooperative and simple games. Alternatively, other interesting axiomatizations could be studied.

The capacity of theoretical studies and applications of the value on the contexts described is high and future research is encouraged. Just to mention here a comment for 3-simple games with abstention, if there is certainty or intuition about the probability of abstention then the $\mathfrak{b}$-power index should be redefined accordingly to the probabilities of each input alternative. This comment extends to arbitrary values of $j$ when there is some evidence on the asymmetry in choosing different levels of approval by the voters.

\section{Acknowledgements}

This research was partially supported by funds from the Spanish Ministry of Economy and Competitiveness (MINECO) and from the European Union (FEDER funds) under grant MTM201566818-P(MINECO/FEDER).

\section{References}

[1] Banzhaf J.F. (1965) Weighted voting doesn't work: a mathematical analysis, Rutgers Law Review 19, 317-343.

[2] Bernardi, G. and Freixas, J. (2019) An axiomatization for two power indices for (3,2)-simple games, International Game Theory Review. 21(2), 1940001 (24 pages).

[3] Carreras, F. (2004) $\alpha$-Decisiveness in simple games, Theory and Decision 56h, 77-91.

[4] Carreras, F. (2005) A decisiveness index for simple games, European Journal of Operational Research 163, 370-387.

[5] Coleman, J.S. (1971) Control of collectivities and the power of a collectivity to act. In: Lieberman, B. (ed.): Social Choice, pp. 269-300. Gordon and Breach, NY.

[6] Dreher, A., Gould, M., Rablen, M.D. and Vreeland, J.R. (2014) The determinants of election to the United Nations Security Council, Public Choice 158, 51-83.

[7] Dubey P. and Shapley L.S. (1979) Mathematical properties of the Banzhaf power index, Mathematics of Operations Research 4, 99-131.

[8] Feltkamp, V. (1995) Alternative axiomatic characterizations of the Shapley and Banzhaf values, Int. Journal of Game Theory 24, 179-186. 
[9] Felsenthal, D.S. and Machover, M. (1997) Ternary voting games, International Journal of Game Theory 26, 335-351.

[10] Felsenthal, D.S. and Machover, M. (1998) The measurament of voting power: theory and practice, problems and paradoxes, Cheltenham: Edward Elgar.

[11] Freixas, J. (2005) Banzhaf measures for games with several levels of approval in the input and output, Annals of Operations Research 137, 45-66.

[12] Freixas J. (2012) Probabilistic power indices for voting rules with abstention, Mathematical Social Sciences 64, 89-99.

[13] Freixas, J. and Zwicker W.S. (2003) Weighted voting, abstention, and multiple levels of approval, Social Choice and Welfare 21, 399-431.

[14] Gould M. and Rablen M.D. (2017) Reform of the United Nations Security Council: equity and efficiency, Public Choice 173, 145-168.

[15] Hsiao C.R. and Raghavan T.E.S. (1992) Monotonicity and dummy free property for multichoice cooperative games, Int. Journal of Game Theory 21, 301-302.

[16] Hsiao C.R. and Raghavan T.E.S. (1993) Shapley value for multichoice cooperative games I, Games and Economic Behavior 5, 240-256.

[17] Owen, G. (1975) Multilinear extensions and the Banzhaf value, Naval Research Logistics Quarterly 22, 741-750.

[18] Parker, C. (2012) The influence relation for ternary voting games, Games and Economic Behavior 75, 867-881.

[19] Penrose L.S. (1946) The elementary statistics of majority voting, Journal of the Royal Statistical Society 109, 53-57.

[20] Pongou R., Tchantcho B. and Diffo Lambo L. (2011) Political influence in multichoice institutions: cyclicity, anonymity and transitivity, Theory and Decision 70, 157-178.

[21] Tchantcho B., Diffo Lambo L., Pongou R., and Mbama Engoulou B. (2008) Voters' power in voting games with abstention: Influence relation and ordinal equivalence of power theories, Games and Economic Behavior 64, 335-350.

[22] Uleri P.V. (2002) On referendum voting in Italy: YES, NO, or non-vote? How Italian parties learned to control referendums, European Journal of Political Research 41, 863-883. 